九州大学学術情報リポジトリ

Kyushu University Institutional Repository

\title{
ESTIMATION OF THE INTEGRATED SQUARED DENSITY DERIVATIVES BY WAVELETS
}

Rao, B. L. S. Prakasa

Indian Statistical Institute

https://doi.org/10.5109/13480

出版情報: Bulletin of informatics and cybernetics. 31 (1), pp.47-65, 1999-03. Research Association of Statistical Sciences

バージョン:

権利関係 : 


\title{
ESTIMATION OF THE INTEGRATED SQUARED DENSITY DERIVATIVES BY WAVELETS
}

By

\author{
B.L.S. Prakasa RA $0^{*}$
}

\begin{abstract}
The problem of estimation of the integral of the squared derivative of a probability density $f$ is considered using wavelet orthonormal bases. For $f$ such that $f^{(d)}$, the $d$-th derivative belongs to the Sobolev space $H_{2}^{s}, s>0$, we obtain the precise asymptotic expression for the mean integrated squared error of the wavelet estimator.
\end{abstract}

Key words and Phrases: Nonparametric estimation of derivative of density; Integrals of squared density derivative; Wavelets.

AMS (1991) Subject classification: Primary 62 G 07.

\section{Introduction}

The motivation for estimation of the functional $I_{d}(f)=\int_{-\infty}^{\infty} f^{(d)^{2}}(x) d x$ where $f$ is a probability density and $f^{(d)}$ is its $d$-th derivative is well known. For instance, the functional $I_{2}(f)$ appears in the asymptotics of the integrated mean squared error of a kernel-type density estimator (cf. Prakasa Rao (1983), p.63). Kernel-type estimation for the functional $I_{d}(f)$ has been investigated recently by Hall and Marron (1987), Bickel and Ritov (1988), Jones and Sheather (1991) and Hall and Wolff (1995) among others. In a recent paper, Birge and Massart (1995) studied estimation of functionals of the type $T(f)=\int_{-\infty}^{\infty} \phi\left(x, f(x), f^{(1)}(x), \ldots, f^{(k)}(x)\right) d x$ where $\phi(x)$ is a smooth function of $k+2$ variables and $f$ belongs to a class of probability densities of smoothness $s$. Birge and Massart (1988) generalized the results on the bounds for the rates of convergence of the mean squared error obtained by Bickel and Ritov (1988) to general functions of the type $T(f)$. The motivation for the estimation of general functionals $T(f)$ comes from the need, for instance, in the selection of bandwidth for density estimation, for the estimation of the Fisher information and for the estimation of Shannon entropy etc.(cf. Prakasa Rao (1983)).

In Prakasa Rao (1996), we have studied nonparametric estimation of the derivative of a density by wavelets and obtained a precise asymptotic expression for the mean integrated squared error following techniques of Masry (1994). Estimation of the integral

\footnotetext{
* Indian Statistical Institute, 7, S.J.S. Sansanwal Marg, New Delhi-110016, India.
} 
of squared density was discussed in Prakasa Rao (1997) by the method of wavelets and a precise asymptotic expression for the mean squared error has been obtained. We now extend these results to the case of the estimation of the functional $I_{d}(f)$.

\section{Introduction to Wavelets}

A wavelet system is an infinite collection of translated and scaled versions of functions $\phi$ and $\psi$ called the scaling function and the primary wavelet function respectively. The function $\phi(x)$ is a solution of the equation

$$
\phi(x)=\sum_{k=-\infty}^{\infty} C_{k} \phi(2 x-k)
$$

with

$$
\int_{-\infty}^{\infty} \phi(x) d x=1
$$

and the function $\psi(x)$ is defined by

$$
\psi(x)=\sum_{k=-\infty}^{\infty}(-1)^{k} C_{-k+1} \phi(2 x-k) . .
$$

Note that the choice of the sequence $\left\{C_{k}\right\}$ determines the wavelet system. It is easy to see that

$$
\sum_{k=-\infty}^{\infty} C_{k}=2
$$

Define

$$
\phi_{j, k}(x)=2^{j / 2} \phi\left(2^{j} x-k\right),-\infty<j, k<\infty
$$

and

$$
\psi_{j, k}(x)=2^{j / 2} \psi\left(2^{j} x-k\right),-\infty<j, k<\infty
$$

Suppose the coefficients $\left\{C_{k}\right\}$ satisfy the condition

$$
\begin{aligned}
\sum_{k=-\infty}^{\infty} C_{k} C_{k+2 \ell} & =2 \text { if } \ell=0 \\
& =0 \text { if } \ell \neq 0 .
\end{aligned}
$$

It is known that, under some additional condition on $\phi$, the collection $\left\{\psi_{j, k},-\infty<\right.$ $j, k<\infty\}$ is an orthonormal basis for $L^{2}(R)$ and $\left\{\phi_{j, k},-\infty<k<\infty\right\}$ is an orthonormal system in $L^{2}(R)$ for each $-\infty<j<\infty$ (cf. Daubechies (1990)).

Definition 2.1. A scaling function $\phi \in C^{(r)}$ is said to be $r$-regular for an integer $r \geq 1$ if for every non-negative integer $\ell \leq r$ and for any integer $k$,

$$
\left|\phi^{(\ell)}(x)\right| \leq c_{k}(1+|x|)^{-k},-\infty<x<\infty
$$

for some $c_{k} \geq 0$ depending only on $k$ where $\phi^{(\ell)}(\cdot)$ denotes the $\ell$-th derivative of $\phi$. 
DEFINITION 2.2. A multiresolution analysis of $L^{2}(R)$ consists of an increasing sequences of closed subspaces $\left\{V_{j}\right\}$ of $L^{2}(R)$ such that

(i) $\bigcap_{j=-\infty}^{\infty} V_{j}=\{0\} ;$
(ii) $\bigcup_{j=-\infty}^{\infty} V_{j}=L^{2}(R)$;

(iii) there is a scaling function $\phi \in V_{0}$ such that

$$
\{\phi(x-k),-\infty<k<\infty\}
$$

is an orthonormal basis for $V_{0}$; and for all $h \in L^{2}(R)$,

(iv) for all $-\infty<k<\infty, h(x) \in V_{0} \Rightarrow h(x-k) \in V_{0}$;

(v) $h(x) \in V_{j} \Rightarrow h(2 x) \in V_{j+1}$.

Mallat (1989) has shown that given any multiresolution analysis, it is possible to derive a function $\psi$ (primary wavelet function) such that for any fixed $j,-\infty<$ $j<\infty$, the family $\left\{\psi_{j, k},-\infty<k<\infty\right\}$ is an orthonormal basis of the orthogonal complement $W_{j}$ of $V_{j}$ in $V_{j+1}$ so that $\left\{\psi_{j k},-\infty<j, k<\infty\right\}$ is an orthonormal basis of $L^{2}(R)$. Conversely, given any compactly supported wavelet system, it gives rise to a multiresolution analysis of $L^{2}(R)$ (cf. Daubechies (1990)). When the scaling function $\phi$ is $r$-regular, the corresponding multiresolution analysis is said to be $r$-regular.

Let $H_{2}^{s}$ denote the space of all functions $g(\cdot)$ in $L^{2}(R)$ whose first $(s-1)$ derivatives are absolutely continuous and define the norm

$$
\|g\|_{H_{2}^{s}}=\sum_{j=0}^{s}\left[\int_{-\infty}^{\infty}\left|g^{(j)}(t)\right|^{2} d t\right]^{1 / 2}
$$

LEMMA 2.1. (Mallat (1989)). Let a multiresolution analysis be $r$-regular. Then, for every $0<s<r$, any function $g \in L^{2}(R)$ belongs to $H_{2}^{s}$ iff

$$
\sum_{\ell=-\infty}^{\infty} e_{\ell}^{2} e^{2 s \ell}<\infty
$$

where $e_{\ell}^{2}=\left\|g-g_{\ell}\right\|_{2}^{2}$ and $g_{\ell}$ is the orthogonal projection of $g$ on $V_{\ell}$.

Remarks: The above introduction is based on Antoniades et al. (1994). For a detailed introduction to wavelets, see Chui (1992) or Daubechies (1992). For a brief survey, see Strang (1989). 


\section{Estimation by the Method of Wavelets}

Suppose $X_{1}, \ldots, X_{n}$ are independent and identically distributed random variables with density $f$. Suppose that $f$ is $d$-times differentiable and that $f^{(d)}$ denotes the $d$-th derivative of $f$. We interpret $f^{(0)}$ as $f$. The problem of interest is the estimation of

$$
I_{d}(f)=\int_{-\infty}^{\infty} f^{(d)^{2}}(x) d x .
$$

Assume that $f^{(d)} \in L^{2}(R)$ and there exist $D_{j} \geq 0, \beta_{j} \geq 0$ such that

$$
\left|f^{(j)}(x)\right| \leq D_{j}|x|^{-\beta_{j}} \text { for }|x| \geq 1,0 \leq j \leq d
$$

where $\beta_{0}>1$.

Consider a multiresolution as discussed in Section 2. Let $\phi$ be the corresponding scaling function. Suppose that the multiresolution is $r$-regular for some $r \geq d$. Then, by definition, $\phi \in C^{(r)}, \phi$ and its derivative $\phi^{(j)}$ up to order $r$ are rapidly decreasing i.e., for every integer $m \geq 1$, there exists a constant $A_{m}>0$ such that

$$
\left|\phi^{(j)}(x)\right| \leq \frac{A_{m}}{(1+|x|)^{m}}, \quad 0 \leq j \leq r .
$$

Let

$$
\phi_{\ell, k}(x)=2^{\ell / 2} \phi\left(2^{\ell} x-k\right),-\infty<k, \ell<\infty
$$

Then

$$
\phi_{\ell, k}^{(j)}(x)=2^{(\ell / 2)+\ell j} \phi^{(j)}\left(2^{\ell} x-k\right), \quad 0 \leq j \leq r
$$

and

$$
\left|\phi_{\ell, k}^{(j)}(x)\right| \leq \frac{2^{(\ell / 2)+\ell j} A_{m}}{(1+|x|)^{m}}, \quad 0 \leq j \leq r .
$$

If $d \geq 1$, then it is clear that

$$
\lim _{|x| \rightarrow \infty} \phi_{\ell, k}^{(j)}(x) f^{(d-j-1)}(x)=0, \quad 0 \leq j \leq d-1
$$

for any fixed $\ell$ and $k$. Let $f_{\ell d}$ be the orthogonal projection of $f^{(d)}$ on $V_{\ell}$. Note that

$$
f_{\ell d}(x)=\sum_{j=-\infty}^{\infty} a_{\ell, j} \phi_{\ell j}(x)
$$

where

$$
\begin{aligned}
a_{\ell j} & =\int_{-\infty}^{\infty} f^{(d)}(u) \phi_{\ell, j}(u) d u \\
& =(-1)^{d} \int_{-\infty}^{\infty} f(u) \phi_{\ell, j}^{(d)}(u) d u
\end{aligned}
$$


by (3.6) for $d \geq 1$. Clearly the equation (3.9) holds for $d=0$. Hence, for all $d \geq 0$,

$$
a_{\ell j}=(-1)^{d} E\left[\phi_{\ell, j}^{(d)}\left(X_{1}\right)\right] \text {. }
$$

Further more

$$
e_{\ell}^{2} \equiv\left\|f^{(d)}-f_{\ell d}\right\|_{2}^{2}=\left\|f^{(d)}\right\|_{2}^{2}-\sum_{k=-\infty}^{\infty} a_{\ell k}^{2} \rightarrow 0 \text { as } \ell \rightarrow \infty
$$

by the properties of a multiresolution decomposition. Here $\|g\|_{p}=\left\{\int_{-\infty}^{\infty}|g|^{p} d x\right\}^{1 / p}, p \geq 1$. Note that

$$
I_{d}(f)=\left\|f^{(d)}\right\|_{2}^{2}
$$

Let

$$
f_{K, \ell, d}(x)=\sum_{k=-K}^{K} a_{\ell k} \phi_{\ell, k}(x)
$$

where $K=K_{n}$ is a sequence of positive integers depending on $\ell=\ell_{n}$ tending to infinity as $n \rightarrow \infty$ and $\ell=\ell_{n} \rightarrow \infty$ as $n \rightarrow \infty$. Note that $f_{K, \ell, d}(x)$ is a truncated projection of $f^{(d)}$ on $V_{\ell}$. Given an i.i.d. sample $X_{1}, \ldots, X_{n}$, let

$$
A_{\ell k}=\frac{1}{n(n-1)} \sum_{i=1, i \neq j}^{n} \sum_{j=1}^{n} \phi_{\ell k}^{(d)}\left(X_{i}\right) \phi_{\ell, k}^{(d)}\left(X_{j}\right)
$$

and we estimate $I_{d}(f)$ by

$$
\hat{I}_{d}(f)=\sum_{k=-K}^{K} A_{\ell k}
$$

Note that

$$
E\left(A_{\ell k}\right)=a_{\ell k}^{2}
$$

and

$$
E\left(\hat{I}_{d}(f)\right)=\sum_{k=-K}^{K} a_{\ell k}^{2}
$$

Observe that

$$
\lim _{\ell \rightarrow \infty} \lim _{K \rightarrow \infty} E\left(\hat{I}_{d}(f)\right)=I_{d}(f)
$$

4. Computation of the Mean Integrated Squared Error for $\hat{I}_{d}(f)$

Let

$$
\begin{aligned}
J_{n}^{2} \equiv E\left|\hat{I}_{d}(f)-I_{d}(f)\right|^{2} & \\
& =\operatorname{var}\left(\hat{I}_{d}(f)\right)+\left(E\left(\hat{I}_{d}(f)\right)-I_{d}(f)\right)^{2} \\
& =\operatorname{var}\left(\hat{I}_{d}(f)\right)+\left(\sum_{k=-K}^{K} a_{\ell k}^{2}-\int_{-\infty}^{\infty} f^{(d)^{2}}(x) d x\right)^{2} \\
& =\operatorname{var}\left(\hat{I}_{d}(f)\right)+\left(\left\|f_{K, \ell, d}\right\|_{2}^{2}-\left\|f^{(d)}\right\|_{2}^{2}\right)^{2} .
\end{aligned}
$$


Note that

$$
f^{(d)}=f^{(d)}-f_{\ell d}+f_{\ell d}-f_{K, \ell, d}+f_{K, \ell, d}
$$

and

$$
\left\|f^{(d)}\right\|_{2}^{2}=\left\|f^{(d)}-f_{\ell d}\right\|_{2}^{2}+\left\|f_{\ell d}-f_{K, \ell, d}\right\|_{2}^{2}+\left\|f_{K, \ell, d}\right\|_{2}^{2}
$$

Hence

$$
\begin{aligned}
\left\|f^{(d)}\right\|_{2}^{2}-\left\|f_{K, \ell, d}\right\|_{2}^{2} & =\left\|f^{(d)}+f_{\ell d}\right\|_{2}^{2}-\left\|f_{\ell d}-f_{K, \ell, d}\right\|_{2}^{2} \\
& =e_{\ell}^{2}+Q_{n}^{2} \equiv B_{n}^{2}
\end{aligned}
$$

where

$$
Q_{n}^{2} \equiv\left\|f_{\ell d}-f_{K, \ell, d}\right\|_{2}^{2} \text { and } e_{\ell}^{2}=\left\|f^{(d)}-f_{\ell d}\right\|_{2}^{2}
$$

Hence

$$
J_{n}^{2}=\operatorname{var}\left(\hat{I}_{d}(f)\right)+\left(e_{\ell}^{2}+Q_{n}^{2}\right)^{2}=\operatorname{var}\left(\hat{I}_{d}(f)\right)+B_{n}^{4} .
$$

Throughout the following discussion, we assume that (A1) the multiresolution analysis given by $\phi$ is $r$-regular where $r \geq 1$ is a positive integer, the function $f^{(d)} \in H_{2}^{s}$ where $0<s<r$ and the function $f$ is of bounded variation on $R$ and (A2) $1<\beta_{0}<$ $(7 / 6)+(d / 3)$.

As a consequence of Lemma 2.1., it follows that

$$
e_{\ell}^{2}=\left\|f^{(d)}-f_{\ell d}\right\|_{2}^{2}=O\left(e^{-2 s \ell}\right) .
$$

Note that

$$
\begin{aligned}
Q_{n}^{2} & =\left\|f_{\ell_{n} d}-f_{K_{n}, \ell_{n}, d}\right\|_{2}^{2} \\
& =\sum_{|j|>K_{n}}\left|a_{\ell_{n}, j}\right|^{2} .
\end{aligned}
$$

But

$$
\begin{aligned}
a_{\ell j} & =(-1)^{d} \int_{-\infty}^{\infty} f(u) \phi_{\ell, j}^{(d)}(u) d u \\
& =(-1)^{d} 2^{\ell\left(\frac{1}{2}+d\right)} \int_{-\infty}^{\infty} \phi^{(d)}\left(2^{\ell} u-j\right) f(u) d u \\
& =(-1)^{d} 2^{\ell\left(\frac{1}{2}+d\right)} \int_{-\infty}^{\infty} \phi^{(d)}(v) f\left(\frac{v+j}{2^{\ell}}\right) 2^{-\ell} d v \\
& =(-1)^{d} 2^{\ell d-(\ell / 2)} \int_{-\infty}^{\infty} \phi^{(d)}(v) f\left(\frac{v+j}{2^{\ell}}\right) d v
\end{aligned}
$$


Hence

$$
\begin{aligned}
\left|a_{\ell j}\right| \leq & 2^{\ell d-(\ell / 2)}\left\{\int_{|v| \leq \frac{|j|}{2}} \phi^{(d)}(v) f\left(\frac{v+j}{2^{\ell}}\right) d v+\int_{|v|>\frac{|j|}{2}} \phi^{(d)}(v) f\left(\frac{v+j}{2^{\ell}}\right) d v\right\} \\
\leq & 2^{\ell d-(\ell / 2)}\left\{\left[\sup _{|v| \leq|j| / 2} f\left(\frac{v+j}{2^{\ell}}\right)\right] \int_{-\infty}^{\infty}\left|\phi^{(d)}(v)\right| d v\right\} \\
& +\left\{\left[\sup _{|v|>|j| / 2}\left|\phi^{(d)}(v)\right|\right] \int_{-\infty}^{\infty} f\left(\frac{v+j}{2^{\ell}}\right) d v\right\} \\
\leq & 2^{\ell d-(\ell / 2)}\left\{\frac{D_{0}}{\left(|j| / 2^{\ell+1}\right)^{\beta 0}}\left\|\phi^{(d)}\right\|_{1}+\frac{A_{m}}{(1+|j| / 2)^{m}} 2^{\ell}\right\} \\
\leq & 2^{\ell d-(\ell / 2)}\left\{\frac{D_{0}\left\|\phi^{(d)}\right\|_{1} 2^{(\ell+1) \beta_{0}}}{|j|^{\beta_{0}}}+\frac{2^{\ell+m}}{|j|^{m}} A_{m}\right\} .
\end{aligned}
$$

Hence

$$
\begin{aligned}
Q_{n}^{2} \leq & 2^{2 \ell_{n} d-\ell_{n}+1}\left\{D_{0}^{2}\left\|\phi^{(d)}\right\|_{1}^{2} 2^{2 \beta_{0}\left(\ell_{n}+1\right)} \sum_{|j|>K_{n}} \frac{1}{|j|^{2 \beta_{0}}}\right\} \\
& +\left\{2^{2\left(\ell_{n}+m\right)} A_{m}^{2} \sum_{|j|>K_{n}} \frac{1}{|j|^{2 m}}\right\} \\
\leq & 2^{2 \ell_{n} d-\ell_{n}+1}\left\{\frac{D_{0}^{2}\left\|\phi^{(d)}\right\|_{1}^{2} 2^{2 \beta_{0}\left(\ell_{n}+1\right)}}{\left(2 \beta_{0}-1\right) K_{n}^{2 \beta_{0}-1}}+\frac{A_{m}^{2}}{(2 m-1)} \frac{2^{2\left(\ell_{n}+m\right)}}{K_{n}^{2 m-1}}\right\}
\end{aligned}
$$

from (3.2) and (3.6) for any integer $m \geq 1$. Let $m>\beta_{0}$. Then

$$
\begin{aligned}
Q_{n}^{2} & \leq 2\left\{\frac{D_{0}^{2}\left\|\phi^{(d)}\right\|_{1}^{2}}{\left(2 \beta_{0}-1\right)} \frac{2^{\ell_{n}(2 d-1)+2 \beta_{0}\left(\ell_{n}+1\right)}}{K_{n}^{2 \beta_{0}-1}}+\frac{A_{m}^{2} 2^{2\left(\ell_{n}+m\right)+\ell_{n}(2 d-1)}}{\left(2 \beta_{0}-1\right) K_{n}^{2 \beta_{0}-1}}\right\} \\
& \leq \frac{2^{\ell_{n}\left\{(2 d-1)+2 \beta_{0}\right\}}}{K_{n}^{2 \beta_{0-1}}} \frac{2^{2 \beta_{0}+1}}{\left(2 \beta_{0}-1\right)} D_{0}^{2}\left\|\phi^{(d)}\right\|_{1}^{2}\left(1+0\left(2^{2 \ell_{n}\left(1-\beta_{0}\right)}\right)\right) \\
& \leq \frac{2^{\ell_{n}\left\{(2 d-1)+2 \beta_{0}\right\}}}{K_{n}^{2 \beta_{0-1}}} \frac{2^{2 \beta_{0}+1}}{\left(2 \beta_{0}-1\right)} D_{0}^{2}\left\|\phi^{(d)}\right\|_{1}^{2}(1+0(1))
\end{aligned}
$$

since $\beta_{0}>1$ and $\ell_{n} \rightarrow \infty$. If

$$
K_{n}=2^{\left\{(2 d-1)+2 \beta_{0}+2 s\right\}\left(\ell_{n} /\left(2 \beta_{0}-1\right)\right)} \log n,
$$

then

$$
\frac{2^{\ell_{n}\left\{(2 d-1)+2 \beta_{0}\right\}}}{K_{n}^{2 \beta_{0}-1}}=\frac{1}{(\log n)^{2 \beta_{0}-1} 2^{2 s \ell_{n}}} \rightarrow 0 \text { as } n \rightarrow \infty
$$

since $\beta_{0}>1$ and $\ell_{n} \rightarrow \infty$ and in fact

$$
Q_{n}^{2}=O\left(2^{-2 s \ell_{n}}\right)
$$


Note that

$$
\begin{aligned}
B_{n}^{2} & =\left\|f^{(d)}-f_{K_{n}, \ell_{n}, d}\right\|^{2}=Q_{n}^{2}+\left\|f^{(d)}-f_{\ell_{n}, d}\right\|_{2}^{2} \\
& =O\left(2^{-2 s \ell_{n}}\right)+O\left(e^{-2 s \ell_{n}}\right)
\end{aligned}
$$

by Lemma 2.1 (cf. Mallat (1989)) and hence

$$
B_{n}^{2}=0\left(2^{-2 s \ell_{n}}\right) .
$$

Observe that

$$
\begin{aligned}
\operatorname{var}\left(\hat{I}_{d}(f)\right) & =\operatorname{var}\left[\sum_{k=-K}^{K} A_{\ell k}\right] \\
& =\sum_{k=-K}^{K} \sum_{k^{\prime}=-K}^{K} \operatorname{cov}\left(A_{\ell k}, A_{\ell k^{\prime}}\right)
\end{aligned}
$$

where $\operatorname{cov}(X, X)$ is interpreted as $\operatorname{var}(X)$. It can be checked that

$$
\begin{aligned}
\operatorname{var}\left(\hat{I}_{d}(f)\right)= & \frac{2}{n(n-1)} \sum_{k=-K}^{K} \sum_{k^{\prime}=-K}^{K}\left(E\left[\phi_{\ell k}^{(d)}\left(X_{1}\right) \phi_{\ell k^{\prime}}^{(d)}\left(X_{1}\right)\right]\right)^{2} \\
& +\frac{4(n-2)}{n(n-1)} \sum_{k=-K}^{K} \sum_{k^{\prime}=-K}^{K} a_{\ell k} a_{\ell k^{\prime}} E\left[\phi_{\ell k}^{(d)}\left(X_{1}\right) \phi_{\ell k^{\prime}}^{(d)}\left(X_{1}\right)\right] \\
& -\frac{(4 n-6)}{n(n-1)} \sum_{k=-K}^{K} \sum_{k^{\prime}=-K}^{K} a_{\ell k}^{2} a_{\ell k^{\prime}}^{2} .
\end{aligned}
$$

This can be seen from the fact that $X_{i}, 1 \leq i \leq n$ are i.i.d. random variables and $E\left(A_{\ell k}\right)=a_{\ell k}^{2}$ following Prakasa Rao (1983), p.270 and the fact that for any $k$ and $k^{\prime}$,

$$
E\left(A_{\ell k} A_{\ell k^{\prime}}\right)=\frac{1}{n^{2}(n-1)^{2}} \sum E\left[\phi_{\ell k}^{(d)}\left(X_{i}\right) \phi_{\ell k^{\prime}}^{(d)}\left(X_{j}\right) \phi_{\ell k}^{(d)}\left(X_{i}^{\prime}\right) \phi_{\ell k^{\prime}}^{(d)}\left(X_{j}^{\prime}\right)\right]
$$

where the summation runs over all $i, j, i^{\prime}, j^{\prime}$ with $1 \leq i, j, i^{\prime}, j^{\prime} \leq n$. Note that

$$
\begin{aligned}
\left(E\left[\phi_{\ell k}^{(d)}\left(X_{1}\right) \phi_{\ell k^{\prime}}^{(d)}\left(X_{1}\right)\right]\right)^{2} & \\
= & \left(\int_{-\infty}^{\infty} \phi_{\ell k}^{(d)}(u) \phi_{\ell k^{\prime}}^{(d)}(u) f(u) d u\right)^{2} \\
& =\left(\int_{-\infty}^{\infty} \phi_{\ell k}^{(d)^{2}}(u) f(u) d u\right)\left(\int_{-\infty}^{\infty} \phi_{\ell k^{\prime}}^{(d)^{2}}(u) f(u) d u\right) \\
& \quad+\int_{-\infty}^{\infty} \int_{-\infty}^{v}\left[\phi_{\ell k}^{(d)}(u) \phi_{\ell k^{\prime}}^{(d)}(v)-\phi_{\ell k}^{(d)}(v) \phi_{\ell k^{\prime}}^{(d)}(u)\right] f(u) f(v) d u d v
\end{aligned}
$$


by the Lagrange identity. Therefore

$$
\begin{aligned}
\sum_{k=-K}^{K} \sum_{k^{\prime}=-K}^{K}\left(E\left[\phi_{\ell k}^{(d)}\left(X_{1}\right) \phi_{\ell k^{\prime}}^{(d)}\left(X_{1}\right)\right]\right)^{2} & \\
= & \sum_{k=-K}^{K} \sum_{k^{\prime}=-K}^{K}\left(\int_{-\infty}^{\infty} \phi_{\ell k}^{(d)^{2}}(u) f(u) d u\right)\left(\int_{-\infty}^{\infty} \phi_{\ell k^{\prime}}^{(d)^{2}}(u) f(u) d u\right) \\
& +\sum_{k=-K}^{K} \sum_{k^{\prime}=-K}^{K}\left\{\int_{-\infty}^{\infty} \int_{-\infty}^{v}\left[\phi_{\ell k}^{(d)}(u) \phi_{\ell k^{\prime}}^{(d)}(v)-\phi_{\ell k}^{(d)}(v) \phi_{\ell k^{\prime}}^{(d)}(u)\right] f(u) f(v) d u d v\right\} \\
= & \sum_{k=-K}^{K}\left(\int_{-\infty}^{\infty} \phi_{\ell k}^{(d)^{2}}(u) f(u) d u\right) \sum_{k^{\prime}=-K}^{K}\left(\int_{-\infty}^{\infty} \phi_{\ell k^{\prime}}^{(d)^{2}}(u) f(u) d u\right) \\
& +\sum_{k=-K}^{K} \sum_{k^{\prime}=-K}^{K} I_{k, k^{\prime}}^{(\ell)}(\mathrm{say}) .
\end{aligned}
$$

Note that

$$
\begin{aligned}
\frac{1}{2^{\ell_{n}} \sum_{j=-K_{n}}^{K_{n}}}\left[\int_{-\infty}^{\infty} \phi_{\ell_{n}, j}^{(d)^{2}}(u) f(u) d u\right]= & \sum_{j=-\infty}^{\infty}\left[\int_{-\infty}^{\infty} \phi_{\ell_{n}, j}^{(d)^{2}}(u) f(u) d u\right] \\
& -\frac{1}{2^{\ell_{n}}} \sum_{|j|>K_{n}} \int_{-\infty}^{\infty} \phi_{\ell_{n}, j}^{(d)^{2}}(u) f(u) d u \\
= & S_{1}+S_{2} \text { (say). }
\end{aligned}
$$

Since $f$ is of bounded variation on $R$ by assumption, it follows that

$$
\begin{aligned}
S_{1}= & \frac{2^{\ell_{n}(1+2 d)}}{2^{\ell_{n}}} \int_{-\infty}^{\infty} \phi^{(d)^{2}}(u)\left\{\frac{1}{2^{\ell_{n}}} \sum_{j=-\infty}^{\infty} f\left(\frac{u+j}{2^{\ell_{n}}}\right)\right\} d u \\
= & 2^{2 \ell_{n} d} \int_{-\infty}^{\infty} \phi^{(d)^{2}}(u)\left\{\frac{1}{2^{\ell_{n}}} \sum_{j=-\infty}^{\infty} f\left(\frac{u+j}{2^{\ell_{n}}}\right)\right\} d u \\
= & 2^{2 \ell_{n} d} \int_{-\infty}^{\infty} \phi^{(d)^{2}}(u)\left\{\int_{-\infty}^{\infty} f(u) d u+O\left(2^{-\ell_{n}}\right)\right\} d u \\
& (\text { by Lemma A.1 of Masry }(1994)) \\
= & 2^{2 \ell_{n} d}\left\{\int_{-\infty}^{\infty} \phi^{(d)^{2}}(v) d v\right\}\left(1+O\left(2^{-\ell_{n}}\right)\right) .
\end{aligned}
$$

Further more

$$
S_{2}=-\frac{1}{2^{\ell_{n}}} \sum_{|j|>K_{n}} E\left[\phi_{\ell_{n}, j}^{(d)^{2}}\left(X_{1}\right)\right] .
$$


But

$$
\begin{aligned}
E\left[\phi_{\ell_{n}, j}^{(d)^{2}}\left(X_{1}\right)\right] & =\int_{-\infty}^{\infty} \phi_{\ell_{n}, j}^{(d)^{2}}(u) f(u) d u \\
& =2^{\ell_{n}(2 d+1)} \int_{-\infty}^{\infty} \phi^{(d)^{2}}\left(2^{\ell} u-j\right) f(u) d u \\
& =2^{2 \ell_{n} d} \int_{-\infty}^{\infty} \phi^{(d)^{2}}(u) f\left(\frac{u+j}{2^{\ell}}\right) d u \\
& \leq 2^{2 \ell_{n} d}\left\{\frac{D_{0}}{\left(|j| / 2^{\ell_{n}+1}\right)^{\beta_{0}}}\left\|\phi^{(d)^{2}}\right\|_{1}+\frac{A_{m}^{2}}{(1+|j| / 2)^{2 m}} 2^{\ell_{n}}\right\} \\
& \leq 2^{2 \ell_{n} d}\left\{\frac{D_{0}\left\|\phi^{(d)^{2}}\right\|_{1} 2^{\left(\ell_{n}+1\right) \beta_{0}}}{|j|^{\beta_{0}}}+\frac{2^{\ell_{n}+2 m} A_{m}^{2}}{|j|^{2 m}}\right\}
\end{aligned}
$$

by methods similar to those used to derive (4.9). Hence

$$
\begin{aligned}
\left|S_{2}\right| \leq & \frac{2^{\ell_{n} d}}{2^{\ell_{n}}}\left\{\frac{D_{0}\left\|\phi^{(d)^{2}}\right\|_{1} 2^{\beta_{0}\left(\ell_{n}+1\right)}}{\left(\beta_{0}-1\right) K_{n}^{\beta_{0}-1}}+\frac{2^{\ell_{n}+2 m} A_{m}^{2}}{(2 m-1) K_{n}^{2 m-1}}\right\} \\
= & \frac{D_{0}\left\|\phi^{(d)^{2}}\right\|_{1}}{\left(\beta_{0}-1\right)} \frac{2^{\beta_{0}\left(\ell_{n}+1\right)+2 \ell_{n} d-\ell_{n}}}{K_{n}^{\beta_{0}-1}} \\
& +\frac{A_{m}^{2}}{\left(2 \beta_{0}-1\right)} \frac{2^{\ell_{n}+2 m+2 \ell_{n} d-\ell_{n}}}{K_{n}^{2 \beta_{0}-1}} \\
= & \frac{2^{\ell_{n}\left(\beta_{0}+2 d-1\right)} 2^{\beta_{0}}}{\left(\beta_{0}-1\right) K_{n}^{\beta_{0}-1}}\left\{\frac{D_{0}\left\|\phi^{(d)^{2}}\right\|_{1}}{\left(\beta_{0}-1\right)}+O\left(2^{\ell_{n}\left(1-\beta_{0}\right)}\right\}\right. \\
= & O\left(\frac{2^{\ell_{n}\left(\beta_{0}+2 d-1\right)}}{K_{n}^{\beta_{0}-1}}\right)
\end{aligned}
$$

for $m>\beta_{0}>1$ as $\ell_{n} \rightarrow \infty$ from (4.23) and (4.24). Let

$$
S_{3}=\frac{1}{2^{\ell_{n}}} \sum_{|j| \leq K_{n}}\left\{(-1)^{d} a_{\ell_{n} j}\right\}^{2} .
$$

Then

$$
\left|S_{3}\right| \leq \frac{1}{2^{\ell_{n}}} \sum_{j=-\infty}^{\infty} a_{\ell_{n} j}^{2} \leq \frac{1}{2^{\ell_{n}}}\left\|f^{(d)}\right\|_{2}^{2} .
$$

Combining the above results, we have

$$
\begin{aligned}
& \frac{1}{2^{\ell_{n}(1+2 d)}} \sum_{k=-K}^{K} \int_{-\infty}^{\infty} \phi_{\ell k}^{(d)^{2}}(u) f(u) d u \\
& =\int_{-\infty}^{\infty} \phi^{(d)^{2}}(v) d v+\frac{1}{2^{\ell_{n}(1+2 d)}} \sum_{k=-K}^{K} a_{\ell_{n} k}^{2}+O\left(\frac{1}{2^{\ell_{n}(1+2 d)}}\right) .
\end{aligned}
$$


Therefore, following the relation (4.20), we have

$$
\begin{aligned}
\frac{1}{2^{2 \ell_{n}(1+2 d)}} \sum_{k=-K}^{K} \sum_{k^{\prime}=-K}^{K}\left(E\left[\phi_{\ell k}^{(d)}\left(X_{1}\right) \phi_{\ell k^{\prime}}^{(d)}\left(X_{1}\right)\right]\right)^{2} \\
=\left\{\int_{-\infty}^{\infty} \phi^{(d)^{2}}(v) d v+\frac{1}{2^{\ell_{n}(1+2 d)}} \sum_{k=-K}^{K} a_{\ell_{n} K}^{2}+O\left(\frac{1}{2^{\ell_{n}(1+2 d)}}\right)\right\} \\
\quad\left\{\int_{-\infty}^{\infty} \phi^{(d)^{2}}(v) d v+\frac{1}{2^{\ell_{n}(1+2 d)}} \sum_{k^{\prime}=-K}^{K} a_{\ell_{n} k}^{2}+O\left(\frac{1}{2^{\ell_{n}(1+2 d)}}\right)\right\} \\
+\frac{1}{2^{2 \ell_{n}(1+2 d)}}\left\{\sum_{k=-K}^{K} \sum_{k^{\prime}=-K}^{K} I_{k, k^{\prime}}^{\left(\ell_{n}\right)}\right\} \\
\quad\left\{\int_{-\infty}^{\infty} \phi^{(d)^{2}}(v) d v\right\}^{2}+\frac{1}{2^{2 \ell_{n}(1+2 d)}} \sum_{k=-K}^{K} \sum_{k^{\prime}=-K}^{K} a_{\ell_{n} k^{\prime}}^{2} a_{\ell_{n} k^{\prime}}^{2}+O\left(\frac{1}{2^{2 \ell_{n}(1+2 d)}}\right) \\
+\frac{1}{2^{2 \ell_{n}(1+2 d)}}\left\{\sum_{k=-K}^{K} \sum_{k^{\prime}=-K}^{K} I_{k, k^{\prime}}^{\left(\ell_{n}\right)}\right\}
\end{aligned}
$$

since

$$
\left|\sum_{k=-K}^{K} a_{\ell k}^{2}\right| \leq\left\|f^{(d)}\right\|_{2}^{2}<\infty
$$

Let us now consider

$$
\begin{aligned}
& E\left[\phi_{\ell k}^{(d)}\left(X_{1}\right) \phi_{\ell k^{\prime}}^{(d)}\left(X_{1}\right)\right] \\
& =E\left[\phi_{\ell k}^{(d)}\left(X_{1}\right)\right] E\left[\phi_{\ell k^{\prime}}^{(d)}\left(X_{1}\right)\right] \\
& \quad+\operatorname{cov}\left(\phi_{\ell k}^{(d)}\left(X_{1}\right), \phi_{\ell k^{\prime}}^{(d)}\left(X_{1}\right)\right) \\
& =\quad a_{\ell k} a_{\ell k^{\prime}}+O\left[\left(\operatorname{var}\left[\phi_{\ell k}^{(d)}\left(X_{1}\right)\right] \operatorname{var}\left[\phi_{\ell k^{\prime}}^{(d)}\left(X_{1}\right)\right]\right)^{1 / 2}\right]
\end{aligned}
$$

uniformly in $k, k^{\prime}$. Note that

$$
\operatorname{var}\left[\phi_{\ell k}^{(d)}\left(X_{1}\right)\right]=\int_{-\infty}^{\infty} \int_{-\infty}^{v} f(u) f(v)\left(\phi_{\ell k}^{(d)}(u)-\phi_{\ell k}^{(d)}(v)\right)^{2} d u d v \equiv J_{k}^{(\ell)}(\text { say })
$$

by the Lagrange identity. Combining the above relations, we have

$$
\begin{aligned}
& \frac{1}{2^{2 \ell_{n}(1+2 d)}} \operatorname{var}\left(\hat{I}_{d}(f)\right) \\
& =\frac{2}{n(n-1)}\left(\left\{\int_{-\infty}^{\infty} \phi^{(d)^{2}}(v) d v\right\}^{2}+\frac{1}{2^{2 \ell_{n}(1+2 d)}} \sum_{k=-K}^{K} \sum_{k^{\prime}=-K}^{K} a_{\ell_{n} k}^{2} a_{\ell_{n} k^{\prime}}^{2}\right. \\
& \left.\quad+O\left(\frac{1}{2^{2 \ell_{n}(1+2 d)}}\right)+\frac{1}{2^{2 \ell_{n}(1+2 d)}} \sum_{k=-K}^{K} \sum_{k^{\prime}=-K}^{K} I_{k, k^{\prime}}^{\left(\ell_{n}\right)}\right)
\end{aligned}
$$




$$
\begin{aligned}
& +\frac{4(n-2)}{n(n-1)} \frac{1}{2^{2 \ell_{n}(1+2 d)}}\left\{\sum_{k=-K}^{K} \sum_{k^{\prime}=-K}^{K} a_{\ell_{n} k}^{2} a_{\ell_{n} k^{\prime}}^{2}+\sum_{k=-K}^{K} \sum_{k^{\prime}=-K}^{K} a_{\ell_{n} k^{\prime}} a_{\ell_{n} k^{\prime}} O\left(\left(J_{k}^{\left(\ell_{n}\right)} J_{k^{\prime}}^{\ell_{n}}\right)^{1 / 2}\right)\right\} \\
& -\frac{(4 n-6)}{n(n-1)} \frac{1}{2^{2 \ell_{n}(1+2 d)}} \sum_{k=-K}^{K} \sum_{k^{\prime}=-K}^{K} a_{\ell_{n} k^{\prime}}^{2} a_{\ell_{n} k^{\prime}}^{2} \\
& \left.=\frac{2}{n(n-1)}\left\{\int_{-\infty}^{\infty} \phi^{(d)^{2}}(v) d v\right\}^{2}+O\left(\frac{1}{2^{2 \ell_{n}(1+2 d)}}\right)+\frac{1}{2^{2 \ell_{n}(1+2 d)}} \sum_{k=-K}^{K} \sum_{k^{\prime}=-K}^{K} I_{k, k^{\prime}}^{\left(\ell_{n}\right)}\right\} \\
& +\frac{4(n-2)}{n(n-1)}\left[\frac{1}{2^{2 \ell_{n}(1+2 d)}} \sum_{k=-K}^{K} \sum_{k^{\prime}=-K}^{K} a_{\ell_{n} k} a_{\ell_{n} k^{\prime}} O\left(\left(J_{k}^{\left(\ell_{n}\right)} J_{k^{\prime}}^{\ell_{n}}\right)^{1 / 2}\right)\right] .
\end{aligned}
$$

Therefore

$$
\begin{aligned}
& \frac{n(n-1)}{2^{2 \ell_{n}(1+2 d)}} \operatorname{var}\left(\hat{I}_{d}(f)\right) \\
& =\left\{\int_{-\infty}^{\infty} \phi^{(d)^{2}}(v) d v\right\}^{2}+O\left(\frac{1}{2^{2 \ell_{n}(1+2 d)}}\right)+\frac{1}{2^{2 \ell_{n}(1+2 d)}} \sum_{k=-K}^{K} \sum_{k^{\prime}=-K}^{K} I_{k, k^{\prime}}^{\left(\ell_{n}\right)} \\
& \quad+\frac{4(n-2)}{2^{2 \ell_{n}(1+2 d)}} \sum_{k=-K}^{K} \sum_{k^{\prime}=-K}^{K} a_{\ell_{n} k} a_{\ell_{n} k^{\prime}} O\left(\left(J_{k}^{\left(\ell_{n}\right)} J_{k^{\prime}}^{\ell_{n}}\right)^{1 / 2}\right)
\end{aligned}
$$

We shall prove later that

$$
\frac{1}{2^{2 \ell_{n}(1+2 d)}} \sum_{k=-K}^{K} \sum_{k^{\prime}=-K}^{K} I_{k, k^{\prime}}^{\left(\ell_{n}\right)}=o(1)
$$

and

$$
\frac{4(n-2)}{2^{2 \ell_{n}(1+2 d)}} \sum_{k=-K}^{K} \sum_{k^{\prime}=-K}^{K} a_{\ell_{n} k} a_{\ell_{n} k^{\prime}} O\left(\left(J_{k}^{\left(\ell_{n}\right)} J_{k^{\prime}}^{\ell_{n}}\right)^{1 / 2}\right)=o(1)
$$

under the condition (A1) .Hence we have the following main result.

TheOREM 4.1. Suppose the conditions (A1) and (A2) hold. Further suppose that $\ell_{n} \rightarrow \infty$ and

$$
K_{n}=2^{\left\{(2 d-1)+2 \beta_{0}+2 s\right\}\left(\ell_{n} /\left(2 \beta_{0}-1\right)\right)} \log n .
$$

Define $\hat{I}_{d}(f)$ as an estimator for $I_{d}(f)$ where $\hat{I}_{d}(f)$ is as given by the equation (3.15). Then

$$
\frac{n(n-1)}{2^{2 \ell_{n}(1+2 d)}} E\left|\hat{I}_{d}(f)-I_{d}(f)\right|^{2} \rightarrow\left\{\int_{-\infty}^{\infty} \phi^{(d)^{2}}(v) d v\right\}^{2}
$$

as $n \rightarrow \infty$.

Proof. Observe that

$$
\frac{n(n-1)}{2^{2 \ell_{n}(1+2 d)}} E\left|\hat{I}_{d}(f)-I_{d}(f)\right|^{2}
$$




$$
\begin{aligned}
& =\frac{n(n-1)}{2^{2 \ell_{n}(1+2 d)}}\left[\operatorname{var}\left(\hat{I}_{d}(f)\right)+B_{n}^{4}\right] \\
& =\frac{n(n-1)}{2^{2 \ell_{n}(1+2 d)}} \operatorname{var}\left(\hat{I}_{d}(f)\right)+\frac{n(n-1)}{2^{2 \ell_{n}(1+2 d)}} O\left(2^{-4 s \ell_{n}}\right) \\
& =\left\{\int_{-\infty}^{\infty} \phi^{(d)^{2}}(v) d v\right\}^{2}+O\left(\frac{1}{2^{2 \ell_{n}(1+2 d)}}\right)+o(1)+\frac{n(n-1)}{2^{2 \ell_{n}(1+2 d)}} O\left(2^{-4 s \ell_{n}}\right)
\end{aligned}
$$

from the relations (4.33) to (4.35). Hence

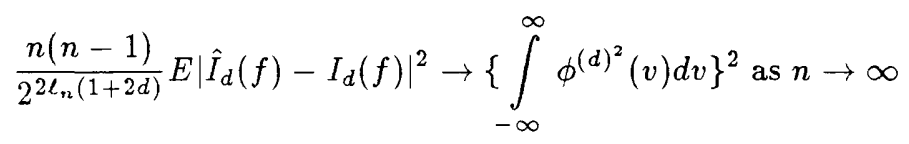

since $\ell_{n} \rightarrow \infty$ as $n \rightarrow \infty$.

Remarks: If $d=0$, then the relation (4.36) reduces to Theorem 3.1 in Prakasa Rao (1997). If $2^{\ell_{n}} \simeq n^{\frac{1}{(2 s+1)}}$, then it follows that the mean squared error $E\left|\hat{I}_{d}(f)-I_{d}(f)\right|^{2}$ is exactly of the order $O\left(\frac{2^{2 \ell n}}{n^{2}}\right)=O\left(n^{\frac{f(d-s)}{(2 \varepsilon+1)}}\right)$. The rate of convergence or the bound on the mean squared error is $O\left(n^{\frac{8(d-s)}{(1+8+1)}}\right)$ for the kernel-type estimator suggested by Bickel and Ritov (1988).

\section{Proof of Equation (4.34)}

Case (i). Suppose $k \neq 0$ and $k^{\prime} \neq 0$. Then

$$
\begin{aligned}
\left|I_{k, k^{\prime}}^{(\ell)}\right| \leq & \int_{-\infty}^{\infty}\left\{\left|\phi_{\ell, k}^{(d)}(u) \phi_{\ell, k}^{(d)}(v)\right|+\left|\phi_{\ell, k^{\prime}}^{(d)}(u) \phi_{\ell, k^{\prime}}^{(d)}(v)\right|\right\} d u d v \\
\leq & 2\left\{\int_{-\infty}^{\infty}\left|\phi_{\ell, k}^{(d)}(u)\right| f(u) d u\right\}\left\{\int_{-\infty}^{\infty}\left|\phi_{\ell, k^{\prime}}^{(d)}(v)\right| f(v) d v\right\} \\
= & 2^{\ell d-(\ell / 2)}\left\{\frac{D_{0}\left\|\phi^{(d)}\right\|_{1} 2^{(\ell+1) \beta_{0}}}{|k|^{\beta_{0}}}+\frac{2^{\ell+m}}{|k|^{m}} A_{m}\right\} \\
& \times 2^{\ell d-(\ell / 2)}\left\{\frac{D_{0}\left\|\phi^{(d)}\right\|_{1} 2^{(\ell+1) \beta_{0}}}{\left|k^{\prime}\right|^{\beta_{0}}}+\frac{2^{\ell+m}}{\left|k^{\prime}\right|^{m}} A_{m}\right\}
\end{aligned}
$$

from the estimate following (4.9)(cf. Prakasa Rao (1996)). Hence

$$
\begin{aligned}
& \sum_{k=-K, k \neq 0}^{K} \sum_{k^{\prime}=-K, k^{\prime} \neq 0}\left|I_{k, k^{\prime}}^{(\ell)}\right| \\
& \leq 2^{2 \ell d-\ell}\left\{\left(D_{0}|| \phi^{(d)} \|_{1} 2^{\beta_{0}(\ell+1)} \sum_{k=-K, k \neq 0}^{K} \frac{1}{|k|^{\beta_{0}}}+2^{\ell+m} A_{m} \sum_{k=-K, k \neq 0}^{K} \frac{1}{|k|^{m}}\right\}^{2}\right.
\end{aligned}
$$




$$
\begin{aligned}
& \leq 2^{2 \ell d-\ell}\left\{2 D_{0}\left\|\phi^{(d)}\right\|_{1} 2^{\beta_{0}(\ell+1)} \int_{1}^{K} \frac{1}{x^{\beta_{0}}} d x+2^{\ell+m} A_{m} \int_{1}^{K} \frac{1}{x^{m}} d x\right\}^{2} \\
& \leq 2^{2 \ell d-\ell}\left\{2 D_{0}\left\|\phi^{(d)}\right\|_{1} 2^{\beta_{0}(\ell+1)} \frac{1-K^{-\beta_{0}+1}}{\beta_{0}-1}+2^{\ell+m} A_{m} \frac{1-K^{-m+1}}{m-1}\right\}^{2} \\
& \leq 2^{2 \ell d-\ell}\left\{C_{1} 2^{2 \ell \beta_{0}}+C_{2} 2^{2 \ell}\right\} \\
& =O\left(2^{\ell\left(2 d-1+2 \beta_{0}\right)}\right)+O\left(2^{2 \ell d+\ell}\right) .
\end{aligned}
$$

Hence

$$
\begin{aligned}
\frac{1}{2^{2 \ell_{n}(1+2 d)}} \sum_{k=-K, k \neq 0}^{K} \sum_{k^{\prime}=-K, k^{\prime} \neq 0}^{K}\left|I_{k, k^{\prime}}^{\ell_{n}}\right| & =O\left(2^{\ell_{n}\left(2 \beta_{0}-3-4 d\right)}\right)+O\left(2^{-\ell_{n}(1+2 d)}\right) \\
& =o(1)
\end{aligned}
$$

provided $\beta_{0}<\frac{3}{2}+2 d$.

Case (ii). Suppose $k=0$ and $k^{\prime} \neq 0$. Note that

$$
\begin{aligned}
a_{\ell 0}=(-1)^{d} \int_{-\infty}^{\infty} \phi_{\ell, 0}^{(d)}(u) f(u) d u & =(-1)^{d} 2^{\ell d-(\ell / 2)} \int_{-\infty}^{\infty} \phi^{(d)}(u) f\left(\frac{u}{2^{\ell}}\right) d u \\
& =O\left(2^{\ell d-(\ell / 2)}\right)
\end{aligned}
$$

by the bounded convergence theorem and the fact that $\left\|\phi^{(d)}\right\|_{1}<\infty$. Now, for $k=0$ and $k^{\prime} \neq 0$,

$$
\left|I_{0, k^{\prime}}^{(\ell)}\right| \leq C_{4}\left\{2^{\ell d-(\ell / 2)}\right\} 2^{\ell d-(\ell / 2)}\left\{\frac{D_{0}\left\|\phi^{(d)}\right\|_{1} 2^{(\ell+1) \beta_{0}}}{\left|k^{\prime}\right|^{\beta_{0}}}+\frac{2^{\ell+m}}{\left|k^{\prime}\right|^{m}} A_{m}\right\}
$$

from (5.3) and the estimate used earlier following Prakasa Rao (1996).It is easy to see that

$$
\left|I_{0, k^{\prime}}^{(\ell)}\right| \leq 2^{2 \ell d-\ell}\left\{C_{5} \frac{2^{\beta_{0} \ell}}{\left|k^{\prime}\right|^{\beta_{0}}}+C_{6} \frac{2^{\ell}}{\left|k^{\prime}\right|^{m}}\right\} .
$$

Therefore

$$
\sum_{k^{\prime}=-K, k^{\prime} \neq 0}^{K}\left|I_{0, k^{\prime}}^{\left(\ell_{n}\right)}\right| \leq C_{7} 2^{\ell_{n}\left(\beta_{0}-1+2 d\right)}+C_{8} 2^{2 \ell_{n} d}
$$

and

$$
\frac{1}{2^{2 \ell_{n}(1+2 d)}} \sum_{k^{\prime}=-K, k^{\prime} \neq 0}^{K}\left|I_{0, k^{\prime}}^{\left(\ell_{n}\right)}\right| \leq C_{7} 2^{\ell_{n}\left(\beta_{0}-3-2 d\right)}+C_{8} 2^{-2 \ell_{n}(1+d)}=o(1)
$$

since $\beta_{0}<3+2 d$.

Case (iii). Suppose $k=0$ and $k^{\prime}=0$. Then

$$
I_{0,0}^{(\ell)} \leq C_{9} 2^{2 \ell d-\ell}
$$

from (5.3) and hence

$$
\frac{1}{2^{2 \ell_{n}(1+2 d)}} I_{0,0}^{\left(\ell_{n}\right)}=O\left(2^{-3 \ell_{n}-2 \ell_{n} d}\right) .
$$


Combining (5.2),(5.6) and (5.7), we obtain that

$$
\frac{1}{2^{2 \ell_{n}(1+2 d)}} \sum_{k=-K}^{K} \sum_{k^{\prime}=-K}^{K}\left|I_{k, k^{\prime}}^{\left(\ell_{n}\right)}\right|=o(1)
$$

proving (4.34).

\section{Proof of Equation (4.35)}

Consider

$$
\begin{aligned}
J_{k}^{(\ell)} & =\int_{-\infty}^{\infty} \int_{-\infty}^{v}\left[\phi_{\ell, k}^{(d)}(u)-\phi_{\ell, k}^{(d)}(v)\right]^{2} f(u) f(v) d u d v \\
& \leq \int_{-\infty}^{\infty} \int_{-\infty}^{\infty}\left[\phi_{\ell, k}^{(d)}(u)-\phi_{\ell, k}^{(d)}(v)\right]^{2} f(u) f(v) d u d v \\
& =\int_{-\infty}^{\infty} \int_{-\infty}^{\infty}\left[\phi_{\ell, k}^{(d)}(u)+\phi_{\ell, k}^{(d)}(v)\right]^{2} f(u) f(v) d u d v \\
& =2 \int_{-\infty}^{\infty} \int_{-\infty}^{\infty} \phi_{\ell, k}^{(d)^{2}} f(u) d u-2\left[\int_{-\infty}^{(d)}(u) \phi_{\ell, k}^{(d)}(v) f(u) f(v) d u d v\right. \\
\leq & \left.2 \int_{-\infty}^{\infty} \phi_{\ell, k}^{(d)} f(u) d u\right]^{2} \\
& -2(u) d u .
\end{aligned}
$$

Case (i). Suppose that $k \neq 0$ and $k^{\prime} \neq 0$. Then

$$
\begin{aligned}
\left|a_{\ell k}\left(J_{k}^{(\ell)}\right)^{1 / 2}\right| \leq & 2^{1 / 2}\left|\int_{-\infty}^{\infty} \phi_{\ell, k}^{(d)} f(u) d u \| \int_{-\infty}^{\infty} \phi_{\ell, k}^{(d)^{2}} f(u) d u\right|^{1 / 2} \\
\leq & 2^{1 / 2} 2^{\ell d-(\ell / 2)}\left\{\frac{D_{0}\left\|\phi^{(d)}\right\|_{1} 2^{(\ell+1) \beta_{0}}}{|k|^{\beta_{0}}}+\frac{2^{\ell+m}}{|k|^{m}} A_{m}\right\} \\
& {\left[2^{\ell d-(\ell / 2)}\left\{\frac{D_{0}\left\|\phi^{(d)}\right\|_{1} 2^{(\ell+1) \beta_{0}}}{|k|^{\beta_{0}}}+\frac{2^{\ell+m}}{|k|^{m}} A_{m}\right\}\right]^{1 / 2} . }
\end{aligned}
$$

The bound given above can be derived by arguments similar to those given in Prakasa Rao (1996). Hence

$$
\left|a_{\ell k}\left(J_{k}^{(\ell)}\right)^{1 / 2}\right| \leq 2^{\ell d-\frac{\ell}{2}}\left\{C_{10} \frac{2^{\beta_{0} \ell}}{|k|^{\beta_{0}}}+C_{11} \frac{2^{\ell}}{|k|^{m}}\right\} 2^{\frac{\ell d}{2}-\frac{\ell}{4}}\left\{C_{12} \frac{2^{\frac{\beta_{0} \ell}{2}}}{|k|^{\beta_{0}}}+C_{13} \frac{2^{\frac{\ell}{2}}}{|k|^{m}}\right\}
$$


by the elementary inequality

$$
(A+B)^{1 / 2} \leq A^{1 / 2}+B^{1 / 2}
$$

for $A>0$ and $B>0$. Therefore

$$
\left|a_{\ell k}\left(J_{k}^{(\ell)}\right)^{1 / 2}\right| \leq 2^{\frac{3 \ell d}{2}-\frac{3 \ell}{4}}\left\{C_{14} \frac{2^{\frac{3 \beta_{0} \ell}{2}}}{|k|^{\frac{3 \beta_{0}}{2}}}+C_{15} \frac{2^{\ell+\frac{\beta_{0} \ell}{2}}}{|k|^{m+\frac{\beta_{0}}{2}}}+C_{16} \frac{2^{\ell \beta_{0}+\frac{\ell}{2}}}{|k|^{\beta_{0}+m}}+C_{17} \frac{2^{\frac{3 \ell}{2}}}{|k|^{2 m}}\right\} .
$$

Hence

$$
\begin{aligned}
& \left|\sum_{k=-K, k \neq 0}^{K} a_{\ell k}\left(J_{k}^{(\ell)}\right)^{1 / 2}\right| \\
& \quad \leq 2^{\frac{3 \ell d}{2}-\frac{3 \ell}{4}}\left\{C_{18} 2^{\frac{3 \beta_{0} \ell}{2}}+C_{19} 2^{\ell+\frac{\beta_{0} \ell}{2}}+C_{20} 2^{\ell \beta_{0}+\frac{l}{2}}+C_{21} 2^{\frac{3 \ell}{2}}\right\} \\
& \leq C_{22} 2^{\frac{3 \ell d}{2}-\frac{3 \ell}{4}} 2^{\frac{3 \beta_{0} \ell}{2}}
\end{aligned}
$$

(since $\beta_{0}>1$ ). Therefore

$$
\left|\sum_{k=-K, k \neq 0}^{K} \sum_{k^{\prime}=-K, k^{\prime} \neq 0}^{K} a_{\ell k}\left(J_{k}^{\left(\ell_{n}\right)}\right)^{1 / 2} a_{\ell k^{\prime}}\left(J_{k}^{\left(\ell_{n}\right)}\right)^{1 / 2}\right| \leq C_{23} 2^{3 \ell d-\frac{3 \ell}{2}} 2^{3 \ell \beta_{0}} .
$$

Hence

$$
\frac{4(n-2)}{2^{2 \ell_{n}(1+2 d)+1}}\left|\sum_{k=-K, k \neq 0}^{K} \sum_{k^{\prime}=-K, k^{\prime} \neq 0}^{K} a_{\ell k}\left(J_{k}^{\left(\ell_{n}\right)}\right)^{1 / 2} a_{\ell k^{\prime}}\left(J_{k^{\prime}}^{\left(\ell_{n}\right)}\right)^{1 / 2}\right| \leq C_{24} n 2^{\ell_{n}\left(3 \beta_{0}-(7 / 2)-d\right)}
$$

since $\beta_{0}<(7 / 6)+(d / 3)$.

Case (ii). Suppose $k=0$ and $k^{\prime} \neq 0$. Since

$$
\begin{aligned}
J_{0}^{\left(\ell_{n}\right)} & \leq 2 \int_{-\infty}^{\infty} \phi_{\ell_{n} 0}^{(d)^{2}} f(u) d u \\
& =2^{\ell_{n}+2 \ell_{n} d+1} 2^{-\ell_{n}} \int_{-\infty}^{\infty} \phi^{(d)^{2}}(u) f\left(\frac{u}{2^{\ell_{n}}}\right) d u \\
& =O\left(2^{2 \ell_{n} d}\right),
\end{aligned}
$$

it follows from (5.3) and (6.7) that

$$
\left|a_{\ell 0}\left(J_{0}^{(\ell)}\right)^{1 / 2}\right| \leq C_{25} 2^{2 \ell d-\frac{\ell}{2}} .
$$

But

$$
\left|\sum_{k=-K, k \neq 0}^{K} a_{\ell k}\left(J_{k}^{(\ell)}\right)^{1 / 2}\right| \leq 2^{\frac{3 \ell d}{2}-\frac{3 \ell}{4}} 2^{\frac{3 \beta_{0} \ell}{2}}
$$


from (6.4). Therefore

$$
\left|\sum_{k^{\prime}=-K, k^{\prime} \neq 0}^{K} a_{\ell 0}\left(J_{0}^{(\ell)}\right)^{1 / 2} a_{\ell k^{\prime}}\left(J_{k^{\prime}}^{(\ell)}\right)^{1 / 2}\right| \leq 2^{3 \ell\left(\frac{\beta_{0}}{2}-\frac{1}{4}+\frac{d}{2}\right.} C_{26} 2^{2 \ell d-(\ell / 2)}
$$

and hence

$$
\begin{aligned}
\frac{4(n-2)}{2^{2 \ell_{n}(1+2 d)+1}}\left|\sum_{k^{\prime}=-K, k^{\prime} \neq 0}^{K} a_{\ell_{n} 0}\left(J_{0}^{\left(\ell_{n}\right)}\right)^{1 / 2} a_{\ell_{n} k^{\prime}}\left(J_{k}^{\prime\left(\ell_{n}\right)}\right)^{1 / 2}\right| & =O\left(n 2^{\ell_{n}\left(\frac{3 \beta_{0}}{2}-\frac{13}{4}-\frac{3 d}{2}\right)}\right) \\
& =o(1)
\end{aligned}
$$

since $\beta_{0}<\frac{13}{6}+\frac{d}{3}$.

Case (iii). Suppose $k=0$ and $k^{\prime}=0$. Note that

$$
\begin{aligned}
a_{\ell O}^{2} J_{0}^{(\ell)} & \leq 2\left[\int_{-\infty}^{\infty} \phi_{\ell, 0}^{(d)} f(u) d u\right]^{2}\left[\int_{-\infty}^{\infty} \phi_{\ell, 0}^{(d)^{2}} f(u) d u\right] \\
& =2\left[2^{\frac{\ell}{2}+\ell d} \int_{-\infty}^{\infty} \phi^{(d)}\left(2^{\ell} u\right) f(u) d u\right]^{2}\left[2^{\ell+2 \ell d} \int_{-\infty}^{\infty} \phi^{(d)^{2}}\left(2^{\ell} u\right) f(u) d u\right] \\
& =2^{2 \ell+4 \ell d+1}\left[\int_{-\infty}^{\infty} \phi^{(d)}\left(2^{\ell} u\right) f(u) d u\right]^{2}\left[\int_{-\infty}^{\infty} \phi^{(d)^{2}}\left(2^{\ell} u\right) f(u) d u\right]
\end{aligned}
$$

and hence

$$
\begin{aligned}
\frac{a_{\ell O}^{2} J_{0}^{(\ell)}}{2^{2 \ell(1+2 d)}} & \leq 2\left(2^{-\ell}\left[\int_{-\infty}^{\infty} \phi^{(d)}(v) f\left(v 2^{-\ell}\right) d v\right]^{2}\right)\left[2^{-\ell} \int_{-\infty}^{\infty} \phi^{(d)^{2}}(v) f\left(v 2^{-\ell}\right) d v\right] \\
& \left.=2^{-3 \ell+1}\left[\int_{-\infty}^{\infty} \phi^{(d)}(v) f\left(v 2^{-\ell}\right) d v\right]^{2}\right]\left[\int_{-\infty}^{\infty} \phi^{(d)^{2}}(v) f\left(v 2^{-\ell}\right) d v\right]
\end{aligned}
$$

which implies that, for $n \geq 2$,

$$
\left.0 \leq \frac{4(n-2)}{2^{2 \ell(1+2 d)}} a_{\ell O}^{2} J_{0}^{(\ell)} \leq 4 n 2^{-3 \ell+1}\left[\int_{-\infty}^{\infty} \phi^{(d)}(v) f\left(v 2^{-\ell}\right) d v\right]^{2}\right]\left[\int_{-\infty}^{\infty} \phi^{(d)^{2}}(v) f\left(v 2^{-\ell}\right) d v\right]
$$

and the expression on the right side tends to zero as $n \rightarrow \infty$ if $\ell=\ell_{n}$ and

$$
\sup _{\ell}\left|\int_{-\infty}^{\infty} \phi^{(d)}(v) f\left(v 2^{-\ell}\right) d v\right|<\infty
$$

and

$$
\sup _{\ell}\left|\int_{-\infty}^{\infty} \phi^{(d)^{2}}(v) f\left(v 2^{-\ell}\right) d v\right|<\infty
$$


Note that

$$
\int_{-\infty}^{\infty} \phi^{(d)}(v) f\left(v 2^{-\ell}\right) d v \rightarrow \int_{-\infty}^{\infty} \phi^{(d)}(v) f(0) d v
$$

and

$$
\int_{-\infty}^{\infty} \phi^{(d)^{2}}(v) f\left(v 2^{-\ell}\right) d v \rightarrow \int_{-\infty}^{\infty} \phi^{(d)^{2}}(v) f(0) d v
$$

as $\ell \rightarrow \infty$ by the bounded convergence theorem and the fact that $\left\|\phi^{(d)}\right\|_{1}<\infty$ and $\left\|\phi^{(d)^{2}}\right\|_{1}<\infty$. Hence

$$
\frac{4(n-2)}{2^{2 \ell(1+2 d)+1}} a_{\ell O}^{2} J_{0}^{(\ell)}=o(1) .
$$

Relations $(6.6),(6.10)$ and $(6.11)$ prove the theorem.

\section{References}

Antoniadis, A. and Carmona, R. (1990): Multiresolution analyses and wavelets for density estimation, Tech. Report, University of California at Irvine.

Antoniadis, A., Gregoire, G. and McKeague, I.W. (1994): Wavelet methods for curve estimation, J. Amer. Statist. Assoc. 89, 1340-1353.

Bickel, P. and Ritov, Y. (1988): Estimating integrated squared density derivatives : sharp best order of convergence estimates, Sankhya A 50, 381-393.

Birge, L. and Massart, P. (1995): Estimation of integral functions of a density, Ann. Statist. 23, 11-29.

Chui, C.K. (1992): An Introduction to Wavelets, Academic Press, Boston.

Daubechies, I. (1990): Orthonormal basis of compactly supported wavelets, Communications on Pure and Applied Math., 49, 906-996.

Hall, P. and Marron, J.S. (1987): Estimation of integrated squared density derivatives, Statist. Prob. Lett. 6, 109-115.

Hall, P. and Wolff, R.C.L. (1995): Estimation of integrals of powers of density derivatives, Statist. Prob. Lett. 24, 105-110.

Jones, M.C. and Sheather, S.J. (1991): Using nonstochastic terms to advantage in kernel-based estimation of integrated square density derivatives, Statist.Prob.Lett. 11, 511-514.

Laurent,B. (1996): Efficient estimation of integral functionals of a density, Ann.Statistics 24, 659-681.

Mallat, S. (1989): Multiresolution approximations and wavelet orthonormal bases of $L^{2}(R)$, Trans. Amer. Math. Soc. 315, 69-87.

Masry, E. (1994): Probability density estimation from dependent observations using wavelet orthonormal bases, Statist. Probab. Lett. 21, 181-194.

Prakasa Rao, B.L.S. (1983): Nonparametric Functional Estimation, Academic Press, Orlando. 
Prakasa Rao, B.L.S. (1996): Nonparametric estimation of the derivatives of a density by the method of wavelets, Bull. Inform. Cyb. 28, 91-100.

Prakasa Rao, B.L.S. (1997): Estimation of integral of square of density by wavelets, Pub. Inst. Stat. Paris 41, 29-48.

Strang, G. (1989): Wavelets and dilation equations : a brief introduction SIAM Review, 31, 614-627.

Received June 1, 1998 